\title{
Ligand Binding Enhances Millisecond Conformational Exchange in Xylanase B2
}

\section{from Streptomyces lividans}

Donald Gagné ${ }^{1, \#,}$, Chitra Narayanan ${ }^{1}$, Nhung Nguyen-Thi ${ }^{1, \&}$, Louise D. Roux ${ }^{1}$, David N. Bernard ${ }^{1}$, Joseph S. Brunzelle $^{2}$, Jean-François Couture ${ }^{3,4,5}$, Pratul K. Agarwal ${ }^{6,7} \&$ Nicolas Doucet $^{1,4,5 *}$

${ }^{1}$ INRS-Institut Armand-Frappier, Université du Québec, 531 Boul. des Prairies, Laval, Québec, H7V 1B7, Canada.

${ }^{2}$ Department of Molecular Pharmacology and Biological Chemistry, Feinberg School of Medicine, Northwestern University, 320 East Superior Street, Chicago, IL, 60611, USA

${ }^{3}$ Ottawa Institute of Systems Biology, Department of Biochemistry, Microbiology and Immunology, University of Ottawa, 451 Smyth Road, Ottawa, Ontario, K1H 8M5, Canada.

${ }^{4}$ PROTEO, the Québec Network for Research on Protein Function, Engineering, and Applications, 1045 Avenue de la Médecine, Université Laval, Québec, QC, G1V 0A6, Canada.

${ }^{5}$ GRASP, the Groupe de Recherche Axé sur la Structure des Protéines, 3649 Promenade Sir William Osler, McGill University, Montréal, QC, H3G 0B1, Canada.

${ }^{6}$ Computational Biology Institute and Computer Science and Mathematics Division, Oak Ridge National Laboratory, 1 Bethel Valley Road, Oak Ridge, TN, 37830, USA.

${ }^{7}$ Department of Biochemistry, Cellular and Molecular Biology, University of Tennessee, Knoxville, TN, 37996, USA.

*Corresponding author: Email: nicolas.doucet@iaf.inrs.ca; Phone: (450) 687-5010, ext. 4212.

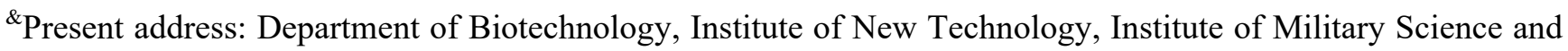
Technology, 17 Hoang Sam, Hanoi, Vietnam.

${ }^{\#}$ Present address: Structural Biology Initiative, CUNY Advanced Science Research Center, 85 St. Nicholas Terrace, New York, NY, 10031, USA. 

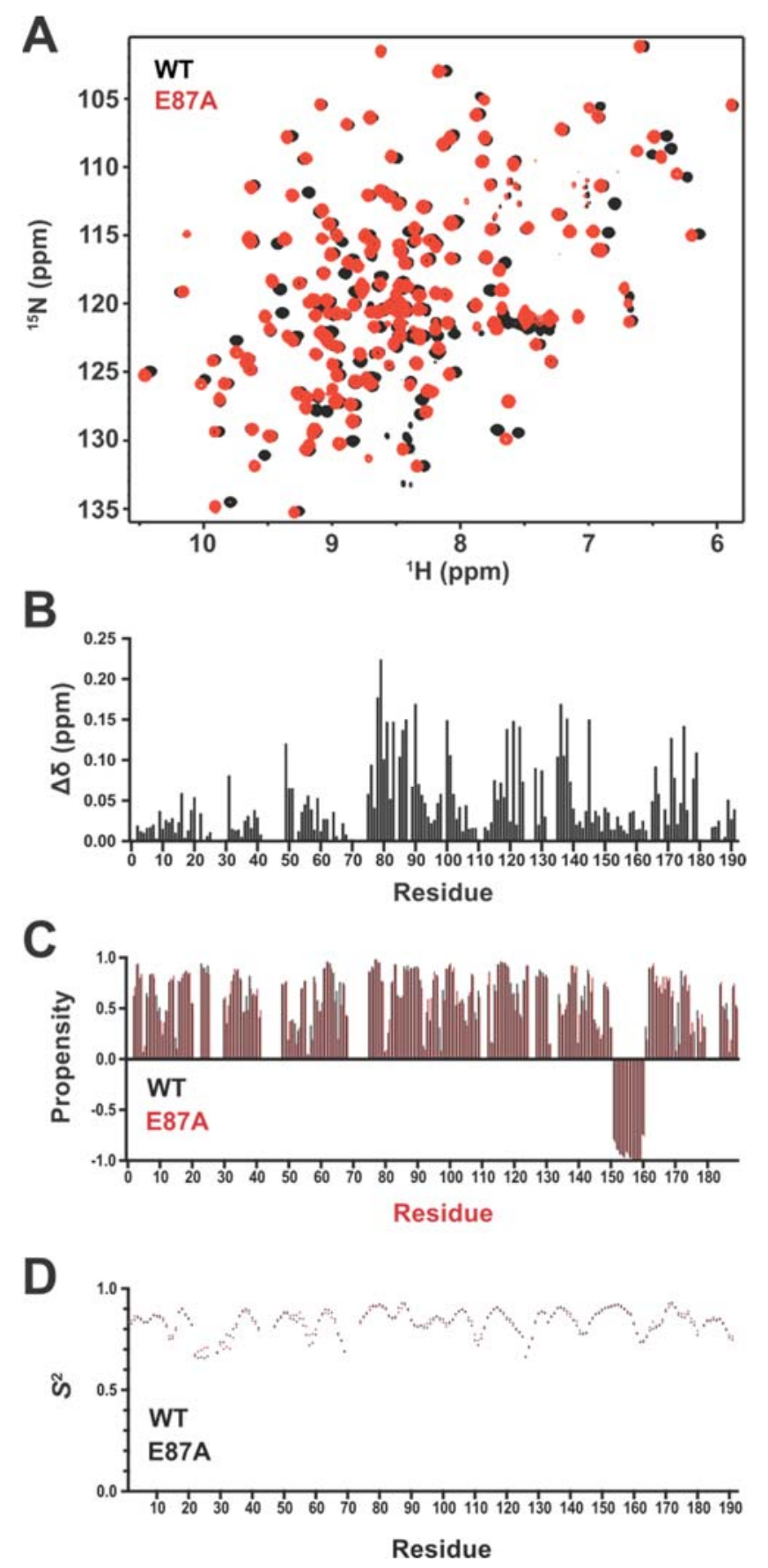

Figure S1. Comparison of structural changes between $\mathrm{X} \operatorname{lnB} 2$ and $\mathrm{X} \ln \mathrm{B} 2-\mathrm{E} 87 \mathrm{~A}$. A) Overlay of ${ }^{1} \mathrm{H}_{-}{ }^{15} \mathrm{~N}$ HSQC spectra of XlnB2 (black) and XlnB2-E87A (red). B) Composite ${ }^{1} \mathrm{H}^{-15} \mathrm{~N}$ chemical shift differences between the E87A mutant and WT XlnB2 (see Experimental Procedures for details). C) Secondary structure propensity for the XlnB2 (black) and XlnB2-E87A (red) predicted using TALOS. D) Order parameters $\left(S^{2}\right)$ for XlnB2 (black) and XlnB2-E87A (red) predicted using TALOS. 

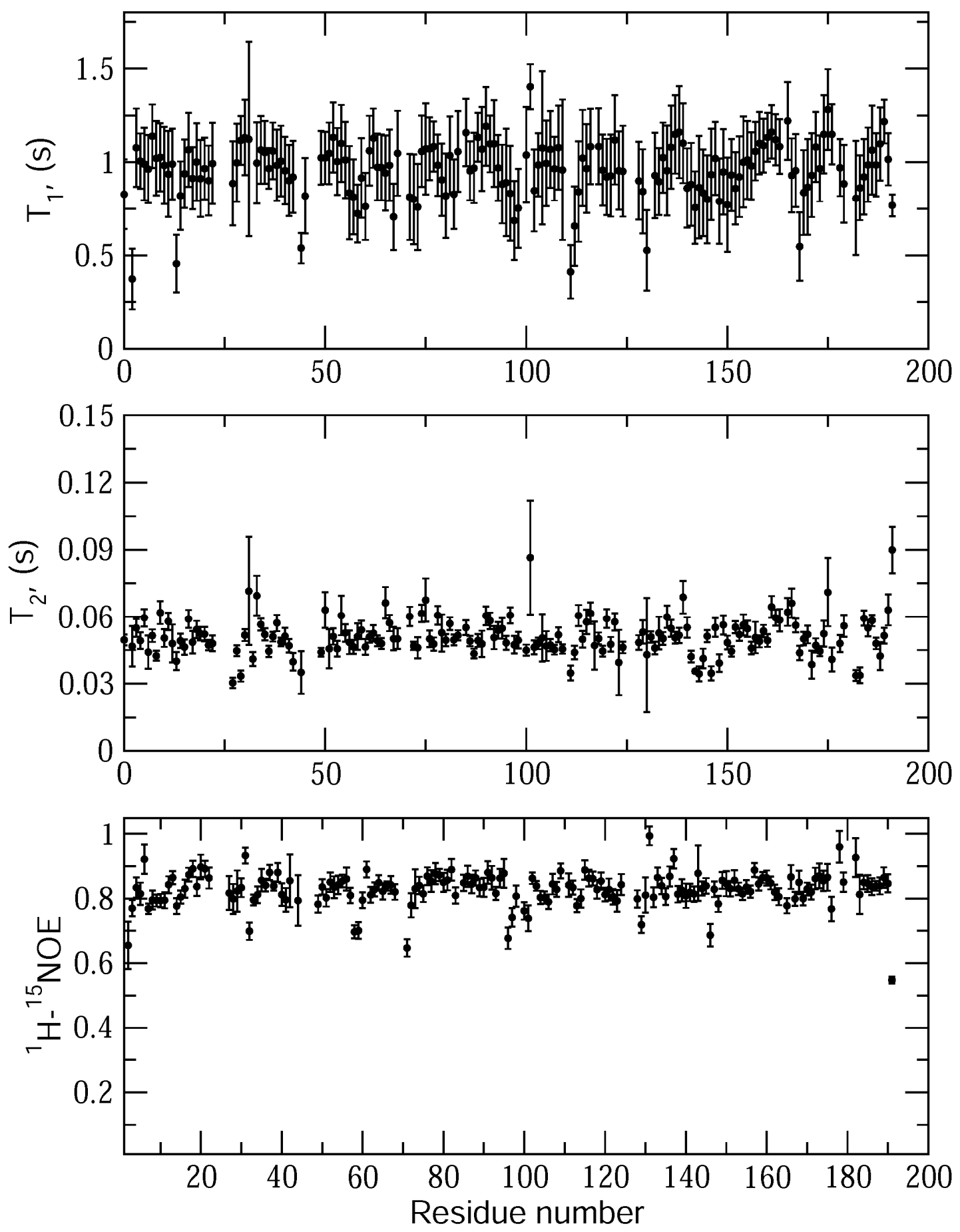

Figure S2. ${ }^{15} \mathrm{~N} T_{1}, T_{2}$ and NOE values for the main chain amides of the apo form of $\mathrm{X} \ln \mathrm{B} 2$. The mean values for all residues are shown as filled circles, with the standard deviation represented using error bars. Two residues ( 32 and 131 were removed from the $T_{1}$ plot due to aberrant values). 

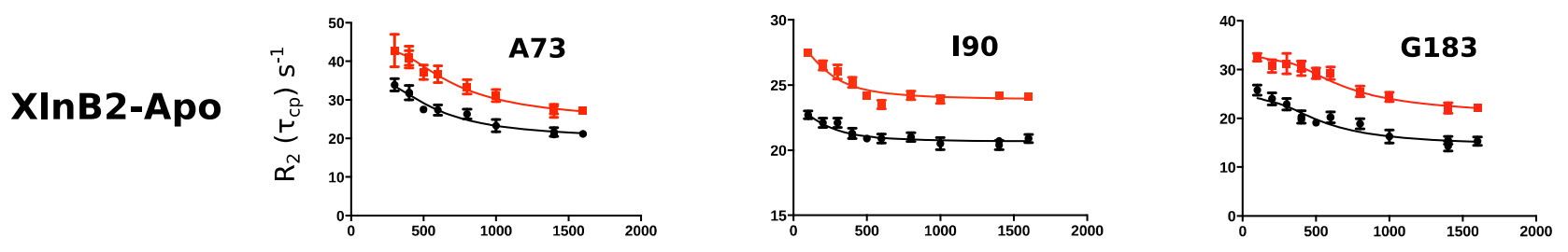

$X \operatorname{lnB2-X2}$
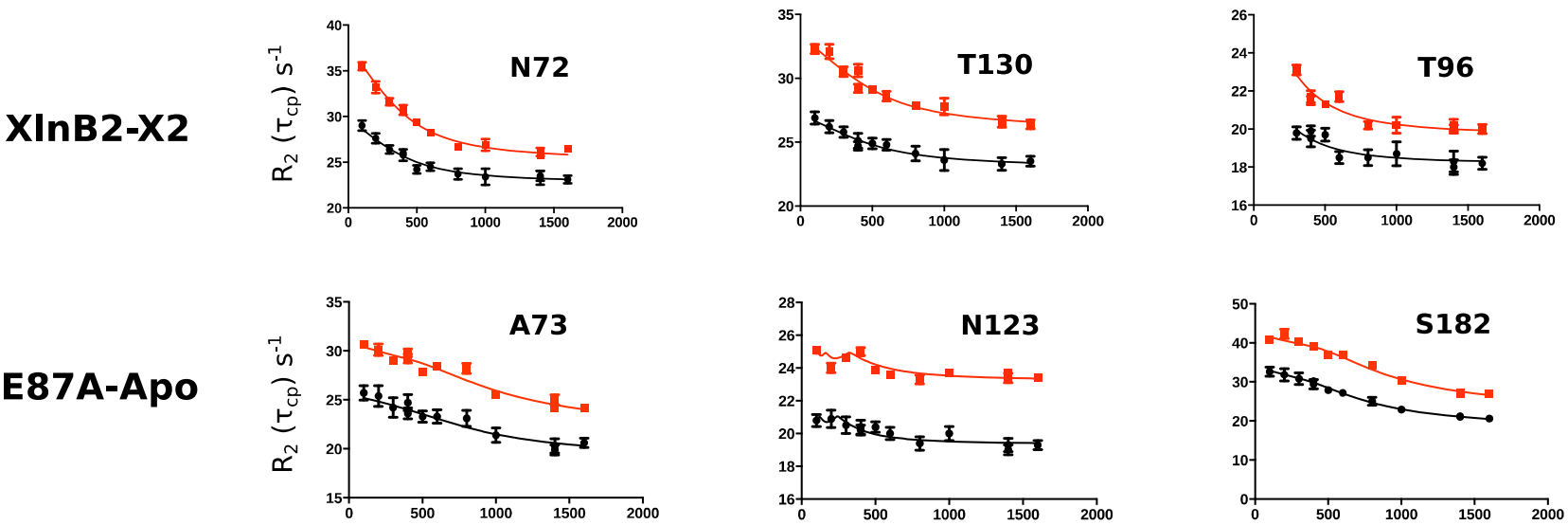

E87A-X2
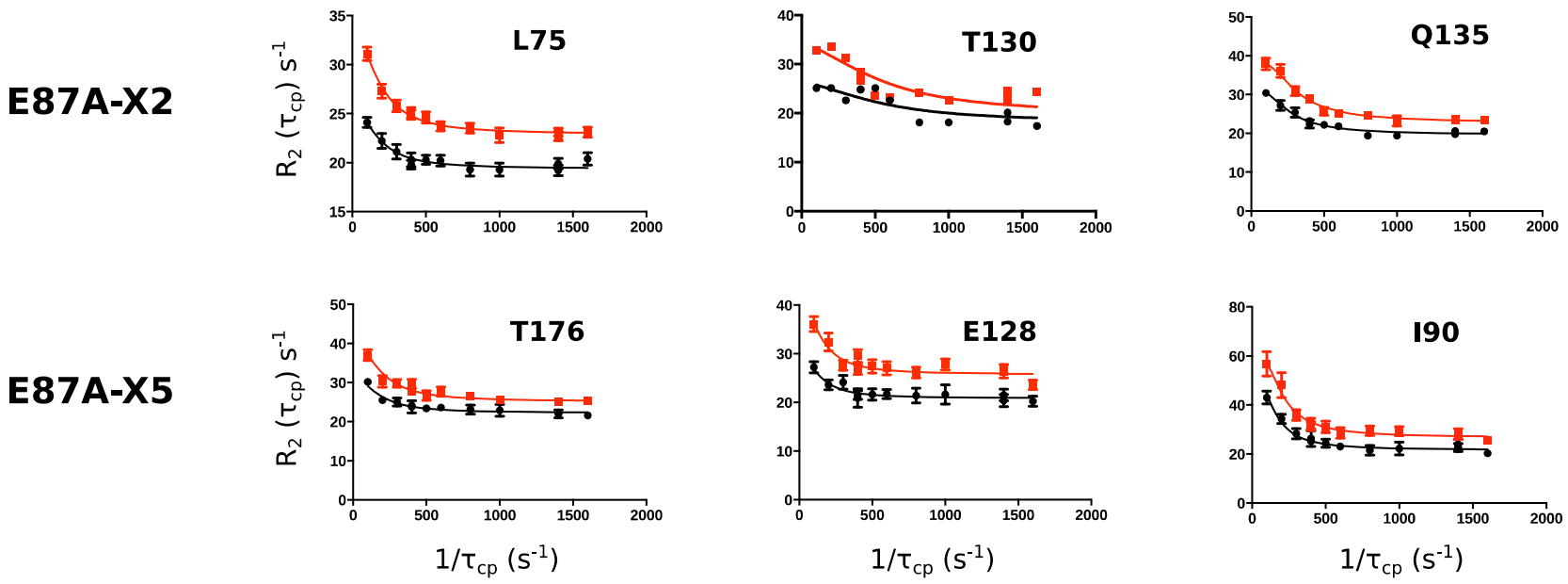

Figure S3. Representative relaxation dispersion curves at $600 \mathrm{MHz}$ (black) and $800 \mathrm{MHz}$ (red) (298K) are shown for residues experiencing ${ }^{1} \mathrm{H}-{ }^{15} \mathrm{~N}$ conformational exchange on the millisecond timescale in the apo and ligand-bound states of $\mathrm{X} \ln \mathrm{B} 2$ and $\mathrm{X} \ln \mathrm{B} 2-\mathrm{E} 87 \mathrm{~A}$. See Experimental Procedures. 

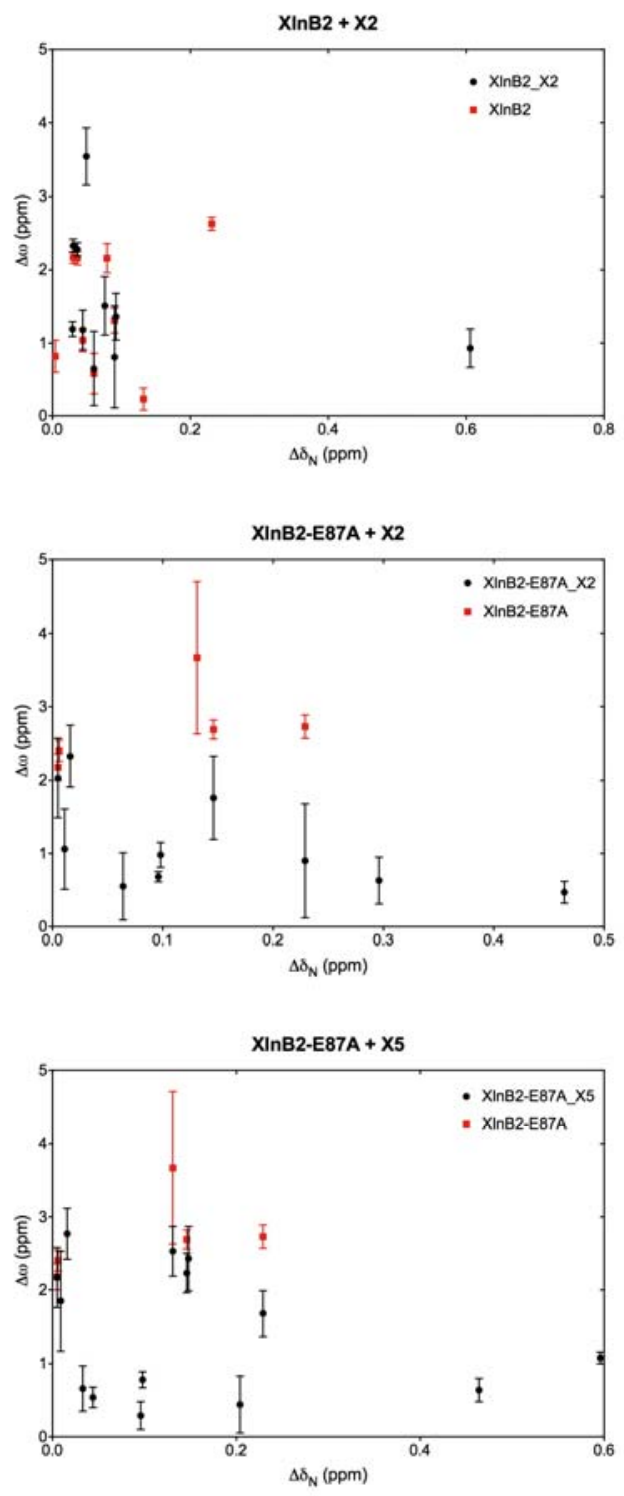

Figure S4. Chemical shift fluctuations (in the nitrogen dimension only, $\Delta \delta_{\mathrm{N}}$ ) observed from titration experiments relative to $\Delta \omega$ values extracted from single-residue dual fits extracted from ${ }^{15} \mathrm{~N}-\mathrm{CPMG}$ relaxation dispersion experiments. This comparison provides information on whether the conformational exchange observed by ${ }^{15} \mathrm{~N}-\mathrm{CPMG}$ results from the exchange between the apo and bound-like conformations in absence of ligand. $\Delta \delta_{\mathrm{N}}$ from XlnB2 titration with $\mathrm{X} 2$ were compared to $\Delta \omega$ values from ${ }^{15} \mathrm{~N}-\mathrm{CPMG}$ experiments performed on both the apo and X2-bound XlnB2 (red squares and black circles in A, respectively). Residues in the binding pocket whose chemical shifts might be influenced by the spatial proximity to the ligand were excluded from this comparison, as only the effect of conformational exchange on $\Delta \delta_{\mathrm{N}}$ was investigated. Similar comparisons were made for the XlnB2 E87A titrations with X2 and X5 (B and C, respectively). Results show no significant correlation between these parameters, suggesting that the chemical shift variations observed upon ligand binding do not exclusively report on the existence of interconverting conformations between a ground and excited state in free $\mathrm{X} \ln \mathrm{B} 2 \mathrm{in}$ solution. 


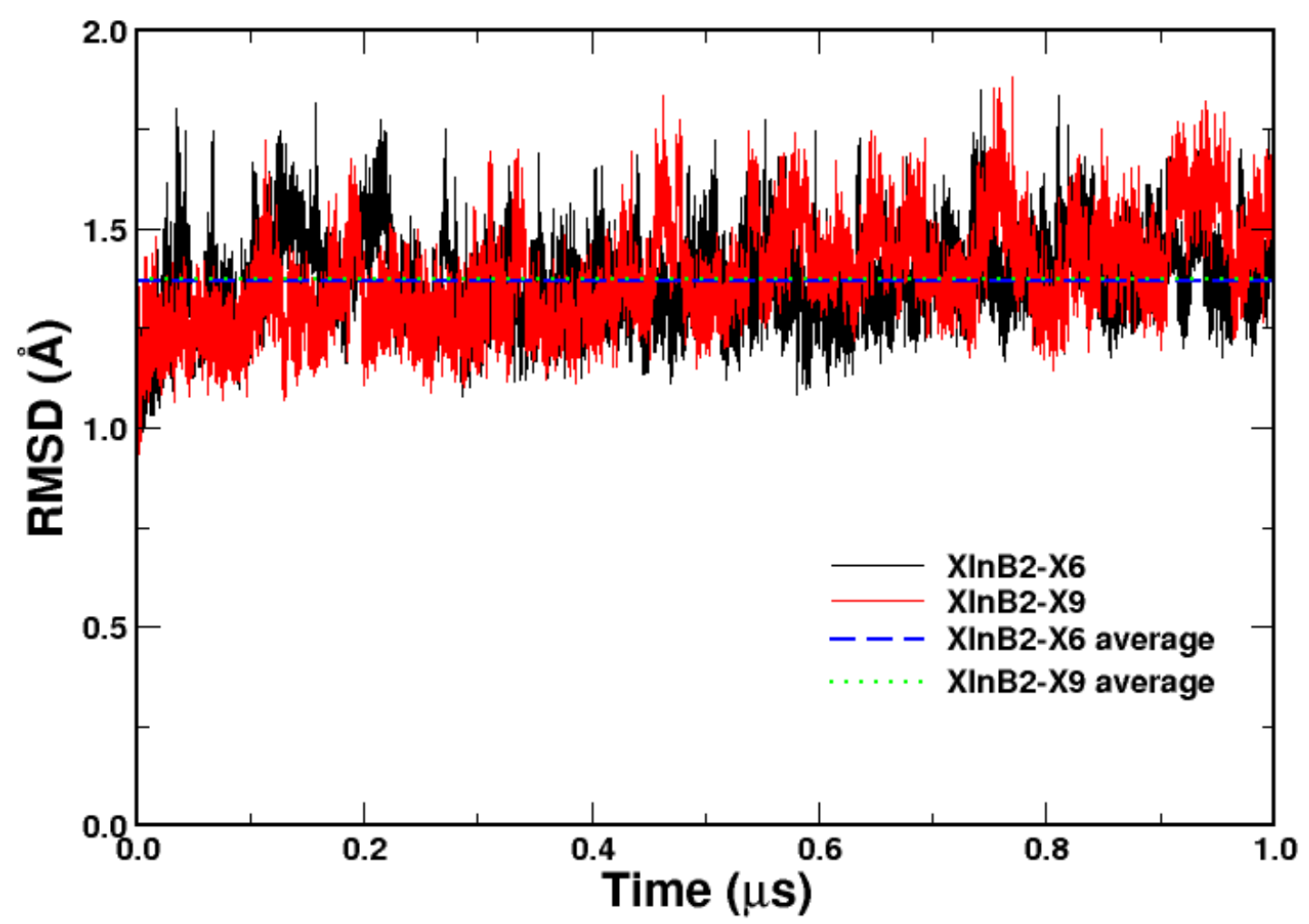

Figure S5. All-atom root mean square deviation (RMSD) as a function of simulation time for XlnB2 in the apo (black) and xylononaose-bound (X9) binary complex (red). RMSDs were calculated using the first structure as the reference structure. Running averages are shown as blue dashed and green dotted lines for $\mathrm{X} \ln \mathrm{B} 2-\mathrm{X} 6$ and $\mathrm{X} \ln \mathrm{B} 2-\mathrm{X} 9$, respectively. 
Table S1. Average enzyme-substrate interaction energies for the interaction between $\mathrm{X} \operatorname{lnB} 2$ and xylohexaose (X6) and xylononaose (X9) substrates. Interaction energies were calculated as the sum of van der Waals and electrostatic energies averaged over all simulation snapshots (see Experimental Procedures).

\begin{tabular}{|c|c|c|c|c|c|}
\hline \multicolumn{3}{|c|}{ XInB2-X6 } & \multicolumn{3}{c|}{ XInB2-X9 } \\
\hline $\begin{array}{c}\text { Enzyme } \\
\text { residue }\end{array}$ & $\begin{array}{c}\text { Substrate } \\
\text { unit }\end{array}$ & $\begin{array}{c}\text { Energy } \\
(\mathrm{kcal} / \mathrm{mol})\end{array}$ & $\begin{array}{c}\text { Enzyme } \\
\text { residue }\end{array}$ & $\begin{array}{c}\text { Substrate } \\
\text { unit }\end{array}$ & $\begin{array}{c}\text { Energy } \\
(\mathrm{kcal} / \mathrm{mol})\end{array}$ \\
\hline 87 & -1 & -5.13 & 87 & -1 & -5.24 \\
\hline 121 & +1 & -3.30 & 20 & -2 & -4.38 \\
\hline 179 & +2 & -3.08 & 121 & -1 & -2.86 \\
\hline 20 & -2 & -3.0 & 97 & +3 & -2.54 \\
\hline 9 & -3 & -2.4 & 179 & +2 & -2.45 \\
\hline 177 & +1 & -1.95 & 89 & -1 & -1.83 \\
\hline 8 & -3 & -1.71 & 179 & +1 & -1.78 \\
\hline 121 & -1 & -1.67 & 78 & -2 & -1.62 \\
\hline 171 & -2 & -1.5 & 125 & -3 & -1.59 \\
\hline 78 & -2 & -1.57 & 20 & -3 & -1.56 \\
\hline
\end{tabular}

